\title{
Metal-poor turnoff and subgiant field stars in the Galaxy
}

\section{I. $R G U$ photometry in a high-latitude field near M101}

\author{
S. Güngör $\mathbf{A k}^{1}$, S. Karaali ${ }^{1}$, and R. Buser ${ }^{2}$ \\ 1 Department of Astronomy and Space Sciences, Istanbul University Observatory, Istanbul, Turkey \\ 2 Astronomisches Institut der Universität Basel, Venusstr. 7, CH-4102 Binningen, Switzerland
}

Received October 29; accepted January 2, 1998

\begin{abstract}
Photographic $R G U$ photometry for about 1800 stars down to a limiting G-magnitude of 19.5 in a highlatitude field $\left((l, b)=\left(101^{\circ},+60^{\circ}\right)\right)$ near the galaxy M101 is investigated. We use an improved variant of the classical three-color method which is based on recent $U B V-R G U-$ photometric transforms and calibrations (Buser 1988; Buser \& Fenkart 1990) and on models of the stellar density distributions of the Galactic population components.

In particular, predictions from the Galactic models of Gilmore \& Wyse (1985) are employed as guides in determining the density functions from the present data, which are then used in turn to derive consistent local luminosity functions. We find that a significant fraction of the intermediate and extreme Population II stars must be (mildly) evolved (rather than main-sequence) stars whose higher-luminosity nature cannot be inferred from their two-color positions only. This conclusion is also consistent with results obtained from combined $R G U-$ photometric and proper motion data in two other Basel fields by Buser \& Chiu (1981a,b), as well as from detailed model-calculations for the larger survey of $R G U$-data in seven high-latitude fields by Buser \& Rong (1995) and Buser et al. (1998a).
\end{abstract}

Key words: Galaxy: structure of - Galaxy: stellar content of - Galaxy: solar neighborhood - stars: population II

\section{Introduction}

The photographic data presented in this paper have been obtained in the context of the re-evaluation program of the Basel $R G U$ three-color photometric high-latitude survey of the Galaxy. The motivation and purpose of this

Send offprint requests to: R. Buser
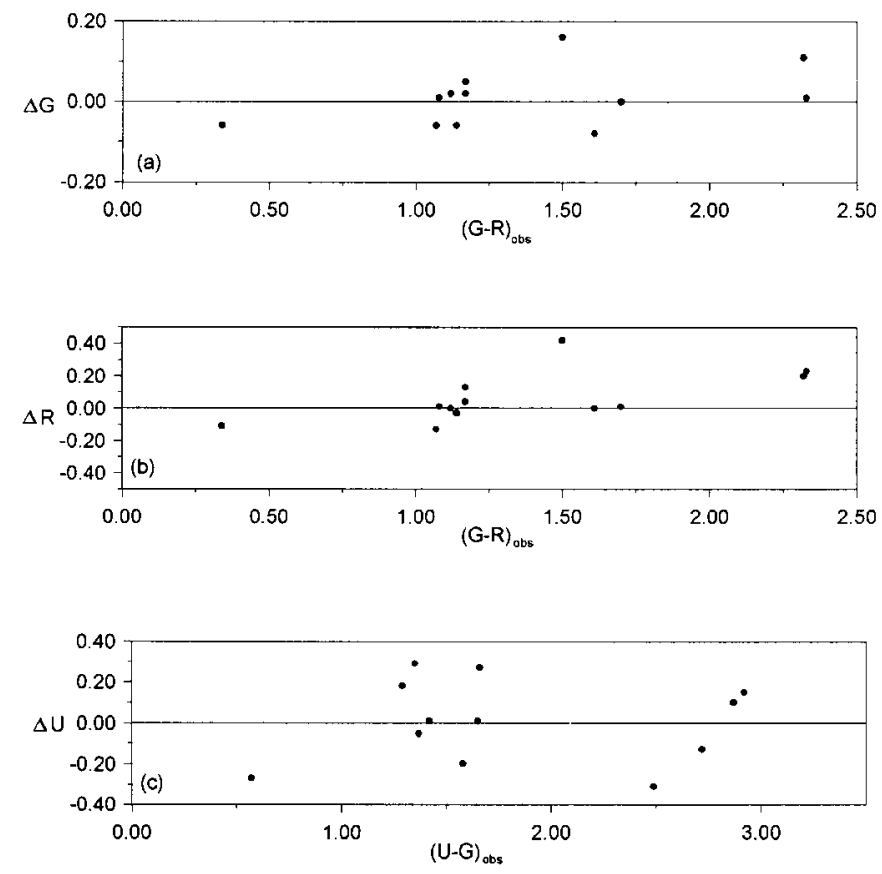

Fig. 1. Residuals between instrumental and standard magnitudes defining the color Eqs. (1) through (5) which have been applied to standardize the present catalog of field data

program are derived from the major fact that a suitable $R G U$ standard system has become available only recently through the advancements in synthetic photometry, and that consequently, the survey plates can be exploited in a more homogeneous and consistent manner, employing more comprehensive calibrations and more adequate methods of analysis than before.

In particular, results based on preliminary data (e.g., Becker 1980; Buser \& Kaeser 1985; Fenkart 1989a-d) were among the first to provide quantitative evidence of a highly flattened intermediate population of faint stars, which is now known as the thick disk component of the 

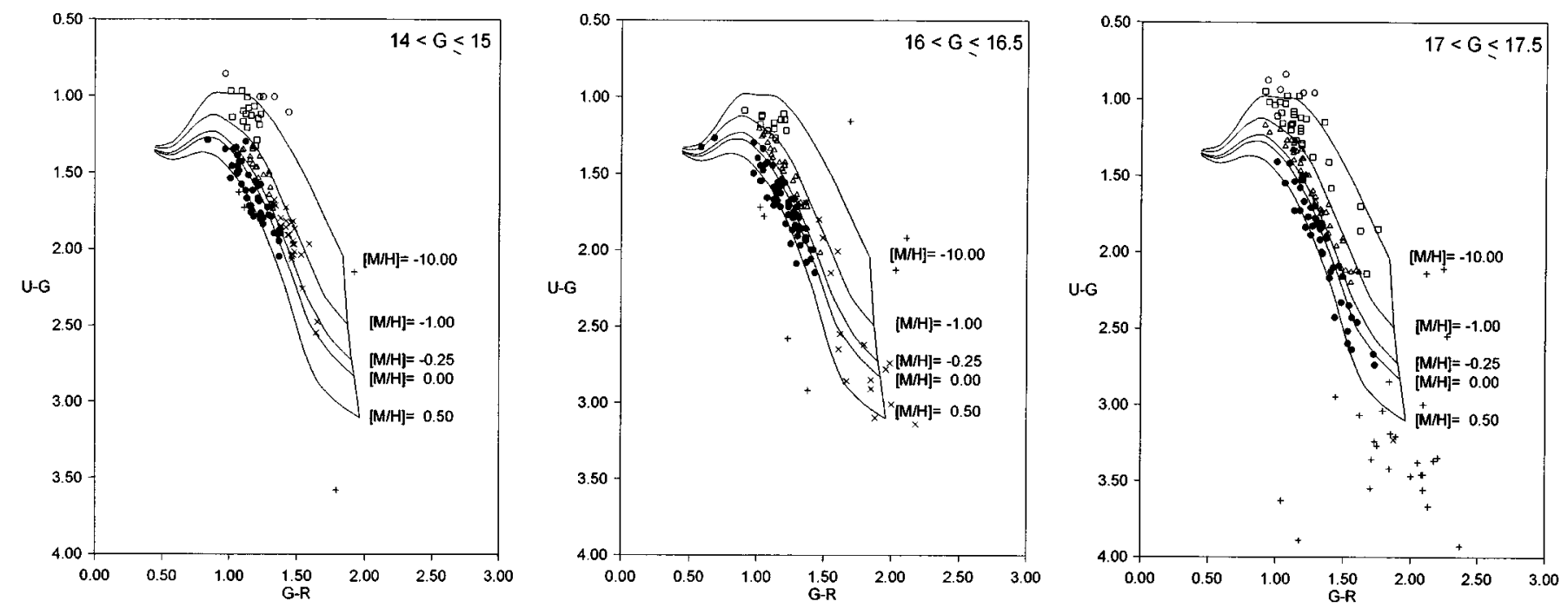

Fig. 2. Sample two-color diagrams for three intervals of successively fainter apparent magnitudes. The superposed grid of isoabundance lines for dwarf stars is based on theoretical model atmosphere calculations (Buser \& Fenkart 1990) and has been used for initially classifying the stars into coarse metallicity classes: $(\bullet)$ population I main sequence; $(\triangle)$ intermediate population II main sequence; $(\square)$ extreme population II main sequence; (०) white objects; $(\times)$ population I late-type red giants; $(+)$ unclassified

Galaxy. It thus became clear that the unparalleled statistical weight and multiplex nature of the Basel survey data immediately called for a definitive evaluation, aimed at a more precise determination of some of the key structural properties of this newly identified population component.

Therefore, the Basel Palomar-Schmidt plates were used for the preparation of a new catalog of homogeneous $R G U$ data for a significant sample of high-latitude fields (Buser et al. 1998b), whence the density and luminosity distributions and, most importantly, also the larger-scale chemical structure of the Galactic thick disk were to be derived.

While the ensemble of the full survey data are being analysed by detailed modelling (cf. Buser \& Rong 1995; Buser et al. 1998a), the data in each individual field will be used to discuss the most important aspects of both the particular field and its analysis in terms of the classical three-color method originally developped by Becker $(1962,1965)$. In the present paper on the Basel field near M101 we shall show how, in the absence of proper motion data, model calculations of the space densities should be used to remedy the notorious failure of the classical, purely photometric method in identifying and properly accounting for the presence of turnoff and subgiant stars among the field populations.

\section{Observations, reduction, and standardization}

In a field of area 2.0 square degrees centered on $(\alpha, \delta(1950.0))=\left(14^{\mathrm{h}} 01^{\mathrm{m}} 24^{\mathrm{s}},+54^{\circ} 35^{\prime}\right)=(l, b)=\left(101^{\circ}\right.$, $\left.+60^{\circ}\right)$, a total of 1806 stars were measured with the Basel
Iris photometer by one of us (S.K.) from $1548^{\prime \prime}$ PalomarSchmidt plates - i.e., 5 plates for each of three $R G U$ colors - taken by Purgathofer and Tammann in 1972. These measurements were reduced to $R G U$ magnitudes using a photoelectric sequence which was established by Sandage and Tammann (1974) and which extends down to limiting $U B V$ magnitudes of $19.85,19.49$, and 18.76 magnitudes, respectively. The $U B V$ data for the calibration stars were converted to the standard $R G U$ system via the transformation equations calculated from a comprehensive grid of detailed flux distributions of spectrophotometric standard stars by Buser (1978a,b).

Calibration curves could then be defined accurately down to limiting $R G U$ magnitudes of 18.07, 19.50, and 21.65 magnitudes, respectively, whence instrumental $R G U$ magnitudes and colors were derived for both the calibration standard and the program stars. Although the number of calibration standard stars is small and the residuals between their instrumental and standard magnitudes as functions of instrumental color show considerable scatter, the resulting color equations in Fig. 1 show the typical behavior expected as resulting from minor mismatches of the photometric passbands behind the instrumental and the standard systems. While the residuals for the two longer-wavelength photometric passbands $(G, R)$ can be well approximated by linear equations, those for the shortwavelength $U$-magnitude feature a characteristic nonlinearity which is due to this near-UV passband's high sensitivity to the strength of the Balmer lines and discontinuity (Buser 1978a, 1988). Consequently, the corresponding color equation has been approximated by a saw-toothlike curve defined by three contiguous linear segments of 

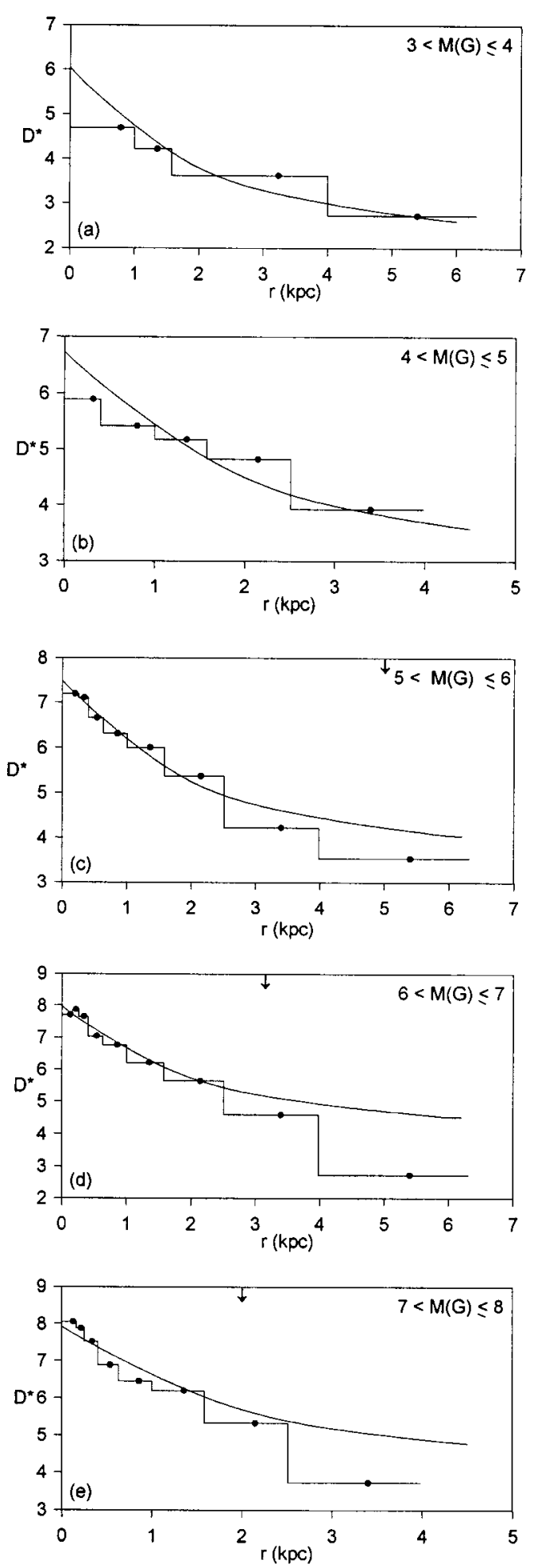

Fig. 3. Histograms of logarithmic space densities as functions of absolute magnitude (panels) for all population components combined. Curves are model density gradients from Gilmore \& Wyse (1985) fitted to the observations at centroid distances (dots) within survey completeness limits (arrows) by least squares. Their intersections with the ordinates $(r=0)$ provide the extrapolated local densities

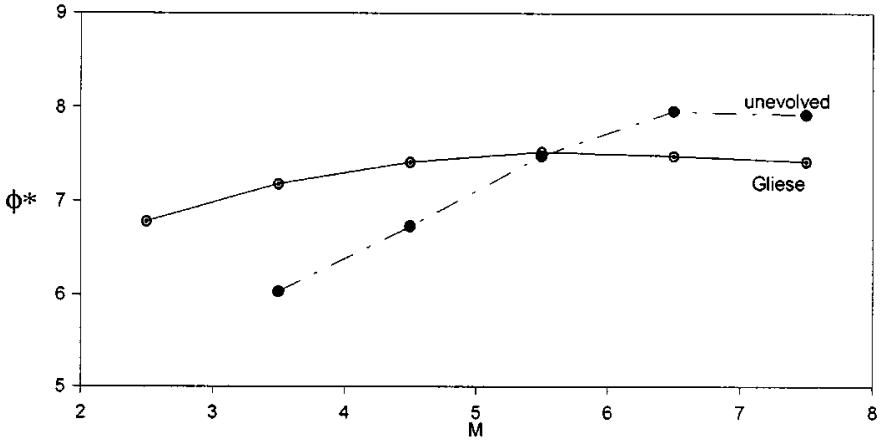

Fig. 4. Luminosity function resulting from observed local densities extrapolated in Fig. 3 (dot-dashed line). Comparison with the luminosity function derived from the Catalog of Nearby Stars by Gliese (1969) indicates that the initial two-color classification of the stars is likely biassed by assuming too many unevolved main sequence stars at faint absolute magnitudes

different slopes. Thus, the following color equations were adopted and applied in generating the final catalog of standard $R G U$ data:

$$
\begin{aligned}
& G_{\mathrm{s}}=G_{\mathrm{obs}}+0.05(G-R)_{\mathrm{obs}}-0.06, \\
& R_{\mathrm{s}}=R_{\mathrm{obs}}+0.17(G-R)_{\mathrm{obs}}-0.17 \text {, } \\
& U_{\mathrm{s}}=U_{\mathrm{obs}}+0.28(U-G)_{\mathrm{obs}}-0.35,(U-G)_{\mathrm{obs}} \leq 1.66 \text {, } \\
& U_{\mathrm{s}}=U_{\mathrm{obs}}-0.36(U-G)_{\mathrm{obs}}+0.60,1.66<(U-G)_{\mathrm{obs}} \\
& \leq 2.49 \text {, } \\
& U_{\mathrm{s}}=U_{\mathrm{obs}}+1.09(U-G)_{\mathrm{obs}}-3.05,2.49<(U-G)_{\mathrm{obs}} \text {. }
\end{aligned}
$$

Full details on the plate journal, the identification charts, and the final photometric data will be published separately in the all-survey catalogs for 14 fields of the Basel high-latitude program (Buser et al. 1998b).

\section{Two-color diagrams and absolute magnitude determination}

A sample of observed $(U-G, G-R)$ two-color distributions as functions of successively fainter apparent $\mathrm{G}$ magnitudes is given in Fig. 2, which shows the classification of the stars into the different categories that determine their subsequent treatment in the statistical analysis, most notably the assignment of absolute magnitudes $\left(M_{G}\right)$ required for the distance and density calculations. The classification is based on the comparison of the observed distributions with the intrinsic loci of the various stellar types provided by the synthetic photometry calibrations. The most important steps of the process are, briefly, as follows.

1. In the absence of systematic errors in the data, unreddened normal population I main sequence stars are expected to scatter uniformly about the theoretical zero-abundance line $([M / H]=0.00)$ (Buser \& Fenkart 

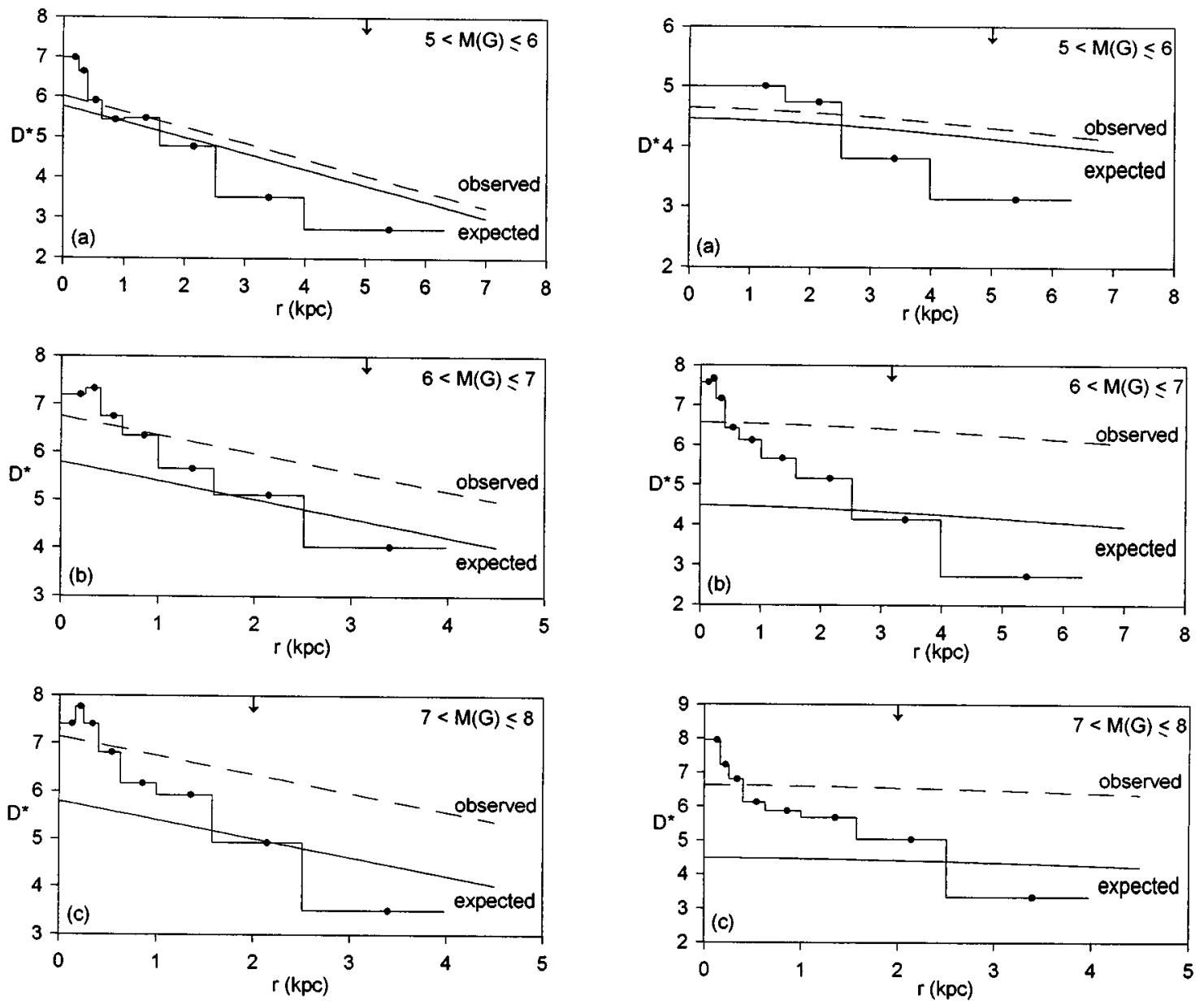

Fig. 5. Histograms of logarithmic space densities as functions of absolute magnitude for the intermediate (left) and the extreme (right) population II components. Solid curves are model density gradients from Gilmore \& Wyse (1985) which, if fitted to the observations as in Fig. 3 (dashed), show that the steeper observed gradients also imply excess extrapolated local densities

Table 1. Logarithmic space densities for Population I stars: $D^{*}=\log D+10$, where $D=N / \Delta V$, and where $N$ is the number of stars in the partial volume $\Delta V_{1,2}=(\pi / 180)^{2}(\square / 3)\left(r_{2}^{3}-r_{1}^{3}\right)$ with field-size $\square$ (in square degrees), limiting distances $r_{1}, r_{2}$, and centroid distance $\bar{r}=\left[\left(r_{1}^{3}+r_{2}^{3}\right) / 2\right]^{1 / 3}$. Distances are in kpc, volumes in $\mathrm{pc}^{3}$

\begin{tabular}{|c|c|c|c|c|c|c|c|c|c|c|c|c|}
\hline & & $M(G)$ & & -4 & & -5 & & -6 & & -7 & & -8 \\
\hline$r_{1}-r_{2}$ & $\bar{r}$ & $\Delta V_{1,2}$ & $N$ & $D^{*}$ & $N$ & $\overline{D^{*}}$ & $N$ & $D^{*}$ & $N$ & $D^{*}$ & $N$ & $D^{*}$ \\
\hline $0.00-0.16$ & 0.13 & $8.01(2)$ & - & & - & & - & & 1 & & - & \\
\hline $0.16-0.25$ & 0.21 & $2.41(3)$ & - & & - & & 2 & 6.79 & 2 & 6.97 & - & \\
\hline $0.25-0.40$ & 0.34 & $9.59(3)$ & - & 4.69 & 1 & 5.39 & 8 & 6.92 & 9 & & 1 & 5.73 \\
\hline $0.40-0.63$ & 0.54 & $3.82(4)$ & - & & - & & 12 & 6.50 & 11 & 6.46 & - & \\
\hline $0.63-1.00$ & 0.86 & $1.52(5)$ & 1 & & 4 & & 27 & 6.25 & 35 & 6.36 & 10 & \\
\hline $1.00-1.59$ & 1.36 & $6.06(5)$ & 1 & 4.22 & 9 & 5.17 & 37 & 5.79 & 43 & 5.85 & 15 & 5.39 \\
\hline $1.59-2.51$ & 2.15 & $2.41(6)$ & 1 & & 13 & 4.73 & 29 & 5.08 & 39 & 5.21 & 4 & 4.22 \\
\hline $2.51-3.98$ & 3.41 & $9.59(6)$ & 4 & 3.62 & 5 & 3.72 & 7 & 3.86 & 14 & 4.16 & - & - \\
\hline $3.98-6.31$ & 5.40 & $3.82(7)$ & 2 & 2.72 & - & - & 6 & 3.20 & - & - & - & - \\
\hline $6.31-10.00$ & 8.55 & $1.52(8)$ & 1 & 1.82 & - & - & - & - & - & - & - & - \\
\hline $10.00-12.59$ & 11.44 & $2.02(8)$ & 2 & 2.00 & - & - & - & - & - & - & - & - \\
\hline
\end{tabular}


Table 2. Logarithmic space densities for Intermediate Population II stars. Symbols as in Table 1

\begin{tabular}{ccccccccccccccc}
\hline & & $M$ & \multicolumn{1}{c}{} & \multicolumn{1}{c}{$2-3$} & \multicolumn{3}{c}{$3-4$} & \multicolumn{3}{c}{$5-6$} & \multicolumn{3}{c}{$6-7$} & \multicolumn{3}{c}{$7-8$} \\
\hline$r_{1}-r_{2}$ & $\bar{r}$ & $\Delta V_{1,2}$ & $N$ & $D^{*}$ & $N$ & $D^{*}$ & $N$ & $D^{*}$ & $N$ & $D^{*}$ & \multicolumn{1}{c}{$N$} & $D^{*}$ \\
\hline $0.00-0.40$ & 0.32 & $1.28(4)$ & - & - & - & - & - & - & - & - & 4 & 6.49 \\
$0.00-0.63$ & 0.50 & $5.10(4)$ & - & - & - & - & - & - & 2 & 5.59 & - & - \\
$0.00-1.00$ & 0.79 & $2.03(3)$ & 1 & 4.69 & 15 & 5.87 & - & - & - & - & - & - \\
$0.00-2.51$ & 1.99 & $3.22(4)$ & - & - & - & - & 16 & 4.70 & - & - & - & - \\
$0.40-0.63$ & 0.54 & $3.82(5)$ & - & - & - & - & - & - & - & - & 8 & 6.32 \\
$0.63-1.00$ & 0.86 & $1.52(5)$ & - & - & - & - & - & - & 4 & 5.42 & 22 & 6.16 \\
$1.00-1.59$ & 1.36 & $6.06(5)$ & - & - & 14 & 5.36 & - & - & 26 & 5.63 & 50 & 5.92 \\
$1.00-2.51$ & 2.03 & $3.02(6)$ & 5 & 4.22 & - & - & - & - & - & - & - & - \\
$1.59-2.51$ & 2.15 & $2.41(6)$ & - & - & 23 & 4.98 & - & - & 30 & 5.10 & 20 & 4.92 \\
$2.51-3.98$ & 3.40 & $9.59(6)$ & 4 & 3.62 & 25 & 4.42 & 3 & 3.50 & 11 & 4.06 & 3 & 3.50 \\
$3.98-6.31$ & 5.40 & $3.82(7)$ & 12 & 3.50 & 6 & 3.20 & 2 & 2.72 & - & - & - & - \\
$6.31-10.00$ & 8.55 & $1.52(8)$ & 2 & 2.12 & - & - & - & - & - & - & - & - \\
\hline
\end{tabular}

Table 3. Logarithmic space densities for Extreme Population II stars. Symbols as in Table 1

\begin{tabular}{|c|c|c|c|c|c|c|c|c|c|c|c|c|}
\hline & & $M(G)$ & \multicolumn{2}{|c|}{$2-3$} & \multicolumn{2}{|c|}{$3-4$} & \multicolumn{2}{|c|}{$5-6$} & \multicolumn{2}{|c|}{$6-7$} & \multicolumn{2}{|c|}{$7-8$} \\
\hline$r_{1}-r_{2}$ & $\bar{r}$ & $\Delta V_{1,2}$ & $\bar{N}$ & $D^{*}$ & $N$ & $\overline{D^{*}}$ & $\bar{N}$ & $D^{*}$ & $N$ & $\overline{D^{*}}$ & $N$ & $D^{*}$ \\
\hline $0.00-1.00$ & 0.79 & $2.03(5)$ & - & - & 7 & 5.54 & - & - & - & - & 7 & 5.54 \\
\hline $0.00-1.59$ & 1.26 & $8.09(5)$ & 6 & 4.87 & - & - & - & - & 21 & 5.41 & - & - \\
\hline $0.00-2.51$ & 1.99 & $3.22(6)$ & - & - & - & - & 7 & 4.34 & - & - & - & - \\
\hline $1.00-1.59$ & 1.36 & $6.06(5)$ & - & - & 6 & 5.00 & - & - & - & - & 28 & 5.66 \\
\hline $1.59-2.51$ & 2.15 & $2.41(6)$ & 10 & 4.62 & 5 & 4.32 & - & - & 33 & 5.14 & 27 & 5.05 \\
\hline $2.51-3.98$ & 3.40 & $9.59(6)$ & 9 & 3.97 & 15 & 4.19 & 6 & 3.80 & 13 & 4.13 & 2 & 3.32 \\
\hline $3.98-6.31$ & 5.40 & $3.82(7)$ & 7 & 3.26 & 12 & 3.50 & 5 & 3.12 & 2 & 2.72 & - & - \\
\hline $6.31-10.00$ & 8.55 & $1.52(8)$ & 2 & 2.12 & - & - & - & - & - & - & - & - \\
\hline
\end{tabular}

Table 4. Logarithmic space densities for all population components combined. Symbols as in Table 1

\begin{tabular}{|c|c|c|c|c|c|c|c|c|c|c|c|c|c|c|c|c|}
\hline & & $M(G)$ & & -3 & & -4 & & -5 & & -6 & & -7 & & -8 & Giants $(M(C$ & $\left.=1^{\mathrm{m}} \pm 1^{\mathrm{m}}\right)$ \\
\hline$r_{1}-r_{2}$ & $\bar{r}$ & $\Delta V_{1,2}$ & $N$ & $D^{*}$ & $N$ & $D^{*}$ & $N$ & $D^{*}$ & $N$ & $D^{*}$ & $N$ & $D^{*}$ & $N$ & $D^{*}$ & $N$ & $D^{*}$ \\
\hline $0.00-0.40$ & 0.32 & $1.28(4)$ & - & - & - & - & 1 & 5.89 & 10 & 6.89 & 12 & 6.97 & 5 & 6.59 & - & - \\
\hline $0.00-1.00$ & 0.79 & $2.03(5)$ & - & - & 23 & 6.05 & - & - & - & - & - & - & - & - & - & \\
\hline $0.00-1.59$ & 1.26 & $8.09(5)$ & 8 & 4.99 & - & - & - & - & - & - & - & - & - & - & - & - \\
\hline $0.00-2.51$ & 1.99 & $3.22(6)$ & - & - & - & - & - & - & - & - & - & - & - & - & - & - \\
\hline $0.40-0.63$ & 0.54 & $3.82(4)$ & - & - & - & - & - & - & 12 & 6.50 & 13 & 6.53 & - & - & - & - \\
\hline $0.40-1.00$ & 0.81 & $1.90(5)$ & - & - & - & - & 4 & 5.32 & - & - & - & - & 47 & 6.39 & - & - \\
\hline $0.63-1.00$ & 0.86 & $1.52(5)$ & - & - & - & - & - & - & 27 & 6.25 & 39 & 6.41 & - & - & - & - \\
\hline $1.00-1.59$ & 1.36 & $6.06(5)$ & - & - & 21 & 5.54 & 9 & 5.17 & 40 & 5.82 & 90 & 6.17 & 93 & 6.19 & - & 4.25 \\
\hline $1.59-2.51$ & 2.51 & $2.41(6)$ & 14 & 4.76 & 29 & 5.08 & 13 & 4.62 & 49 & 5.31 & 102 & 5.63 & - & - & 6 & \\
\hline $1.59-3.98$ & 3.22 & $1.20(7)$ & - & - & - & - & - & - & - & - & - & - & 56 & 4.67 & - & - \\
\hline $2.51-3.98$ & 3.40 & $9.59(6)$ & 13 & 4.13 & 44 & 4.66 & 8 & 3.86 & 16 & 4.22 & - & - & - & - & 17 & \\
\hline $2.51-6.31$ & 5.11 & $4.78(7)$ & - & - & - & - & - & - & - & - & 40 & 3.92 & - & - & - & - \\
\hline $3.98-6.31$ & 5.40 & $3.82(7)$ & 21 & 3.74 & 20 & 3.72 & - & - & 13 & 3.53 & - & - & - & - & 28 & 3.87 \\
\hline $6.31-10.00$ & 8.55 & $1.52(8)$ & 4 & 2.42 & 1 & 1.82 & 1 & 1.82 & - & - & - & - & - & - & 39 & 3.41 \\
\hline $10.00-12.59$ & 11.44 & $2.02(8)$ & - & - & 2 & 2.00 & - & - & - & - & - & - & - & - & 15 & 2.87 \\
\hline $12.59-15.85$ & 14.40 & $4.03(8)$ & - & - & - & - & - & - & - & - & - & - & - & - & 25 & 2.79 \\
\hline $15.85-19.95$ & 18.13 & $8.05(8)$ & - & - & - & - & - & - & - & - & - & - & - & - & $\odot^{1}=$ & $\begin{array}{c}- \\
=7.10\end{array}$ \\
\hline
\end{tabular}



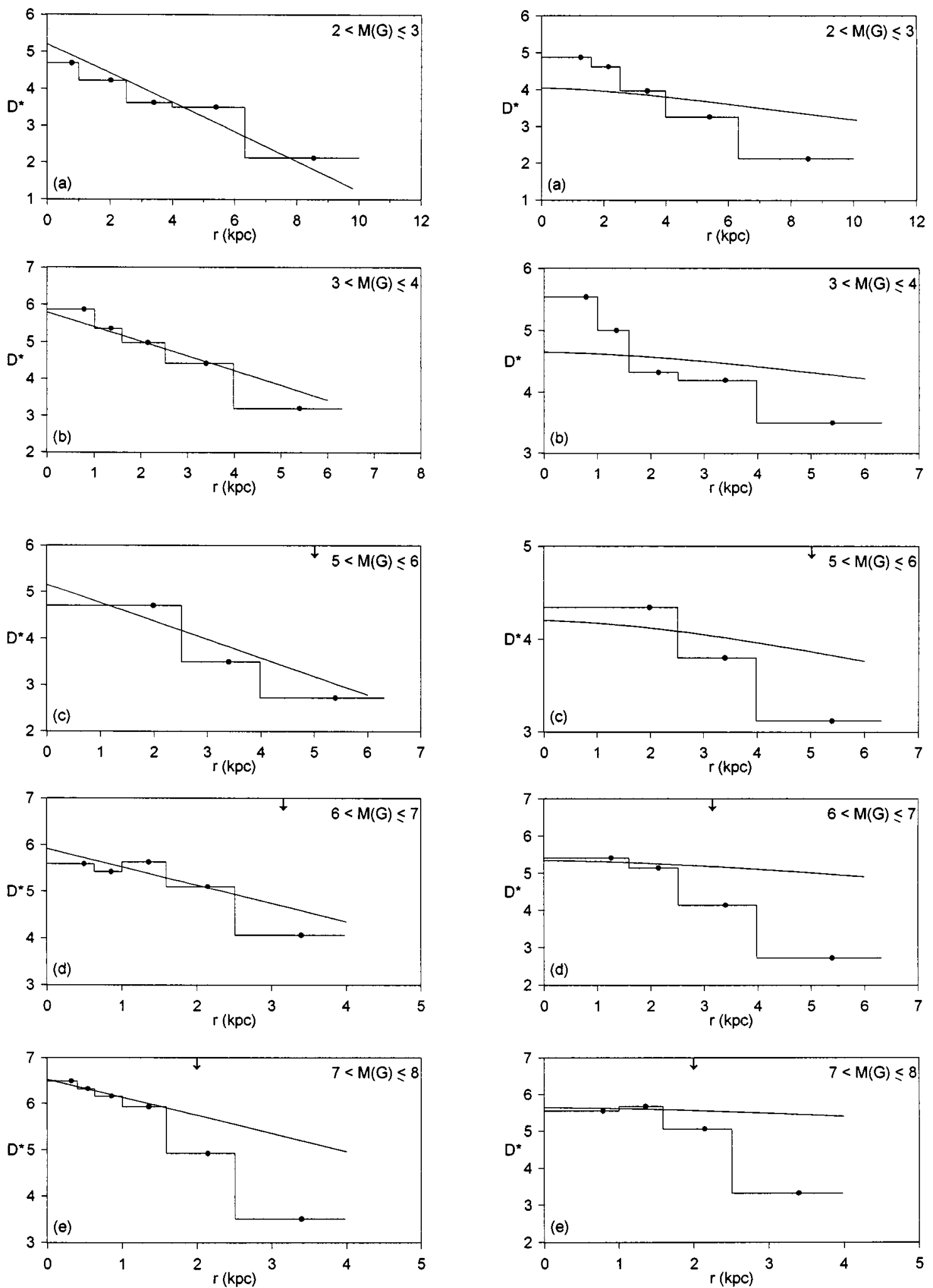

Fig. 6. Histograms of logarithmic space densities as functions of absolute magnitude for the intermediate (left) and the extreme (right) population II components, after assignment of turnoff- and subgiant luminosities $\left(2<M_{G} \leq 4\right)$ to a selected number of lower-metallicity class stars initially assumed to be on the main sequence $\left(5<M_{G} \leq 8\right)$. Note that, in general, the gradients now agree better with the model fits (curves), as do the corresponding extrapolated local densities (see the text) 
1990, hereafter BF90) throughout their observed twocolor ranges and with a dispersion in metallicity of $\sigma_{[M / H]} \sim 0.3$ dex. Thus, the observed distributions of probable normal main sequence stars (full dots) suggest that in this field, interstellar foreground reddening is absent (i.e., $E_{G-R}=0.00$ ), and an absolute magnitude can be assigned to each star by entering its observed $G-R$ in the color-magnitude diagram (hereafter CMD) given in Fig. 7 of BF90 and reading $M_{G}$ off the appropriate standard main sequence for thin-disk dwarfs $(\langle[M / H]\rangle=0.00)$.

2. Most other objects within or near the limits of the grid of theoretical iso-abundance lines for dwarf stars cannot be classified unambiguously from their two-color positions only. In particular, metallicity and luminosity effects on both the $U-G$ and the $G-R$ colors are hardly separable for field stars in the color range $\sim 0.8<G-R<1.5$ (cf. Buser 1978a (Fig. 7), Buser 1982 (Fig. 6), BF90 (Fig. 1 and Table 4)). This means that, e.g., a star's UV-excess, $\delta_{U-G}$, relative to the solar-abundance main sequence may be due to either its advanced evolutionary stage (F-G-type subgiant or G-K-type red giant) or its lower metallicity (intermediate or extreme population II type), or both. Because the assignment of an absolute $M_{G}$ magnitude to an individual star critically depends on its previous assignment of evolutionary stage and population type, we here adopt the following iterative procedure, suggested by BF90.

3.1 The grid of theoretical iso-abundance lines is used to provisionally categorize the objects into two lowermetallicity population types, as follows: stars within the metallicity ranges $-0.25 \geq[M / H]>-1.00$ or $-1.00 \geq[M / H]>-10.00$ are considered (unevolved) main sequence dwarfs of the intermediate (open triangles) or the extreme population II (open squares), respectively, and their absolute $M_{G}$ magnitudes are then given by the corresponding theoretical main sequences (for $\langle[M / H]\rangle=-0.76$ ["thick disk"] or $\langle[M / H]\rangle=$ -1.76 ["halo"]) in the CMD by BF90.

If a discontinuity is apparent in the above two-color distributions near $U-G \sim 1.8-2$, the stars redder than this gap are considered normal red giants (crosses) (Becker 1962, Becker \& Fang 1982). Similarly, stars outside the theoretical grid for dwarfs $(U-G>3.0)$, but whose colors match the predicted synthetic loci for normal population I red giants (Buser 1978a), are classified as such. In either case, these stars are assigned absolute magnitudes $\left\langle M_{G}\right\rangle=1.0$ (Buser 1981).

Objects far outside the theoretical calibrations in the two-color diagrams are either classified as "white objects" (open circles), or are left unclassified (plus signs); both these categories are excluded from the subsequent analysis of the density and luminosity functions.
3.2 Based on the above absolute magnitude determinations, density functions are derived for each of three population types (i.e., main sequence stars of population I, intermediate population II, and extreme population II, respectively), plus their combination. Using a least-squares method, best-fitting model density gradients predicted by Gilmore \& Wyse $(1985)^{1}$ are then matched to the observed density profiles in order to extrapolate hence the local stellar space densities as functions of absolute $M_{G}$ magnitude (del Rio \& Fenkart 1987; Fenkart \& Karaali 1987; Fenkart 1989a-d).

Comparison with the local luminosity function based on the trigonometric parallax catalog of nearby stars by Gliese (1969) then provides the crucial consistency check: if the two disagree substantially and systematically, an iteration cycle is started by reassigning permissible alternative absolute magnitudes to an appropriately chosen number of suitable stars in such a way as to assimilate the resulting local luminosity function to the Gliese standard ${ }^{2}$.

\section{Density and luminosity functions}

The procedure described in the previous Sect. 3 is illustrated in Figs. 3-5, where the density functions (histograms) for the different population types are given for stars of different absolute magnitudes. In each panel, the observed densities are represented by model curves (i.e., density gradients) which were calculated from the density laws adopted for the corresponding population components by Gilmore \& Wyse (1985) and which were then fitted to the data at the centroid distances of their corresponding volumes (full dots) by least-squares. Note that the intersections of these fits with the ordinates provide the estimates of the local luminosity function extrapolated from the observations.

Results of the original trial are shown for the density profiles summed over all three population components in Fig. 3. It is gratifying to see that for the full range of absolute magnitudes and within the completeness limits of the survey (indicated by the vertical arrows) there is good qualitative agreement between the observed and the model-predicted density gradients. However, the corresponding extrapolated local densities (i.e., at $r=0$ ) yield a luminosity function which is significantly different from the Gliese standard, as displayed in Fig. 4. Obviously,

${ }^{1}$ Note that these particular models just give the scale length and density law but do not include the luminosity function.

${ }^{2}$ In their detailed, fully computerized analysis of the same data, Buser et al. (1998a) also employ the luminosity function derived more recently by Wielen et al. (1983). Results show that the differences with the 1969 Gliese standard used in the present, rather coarse analysis do not, however, put into question the basic robustness of the main conclusions presented here. 

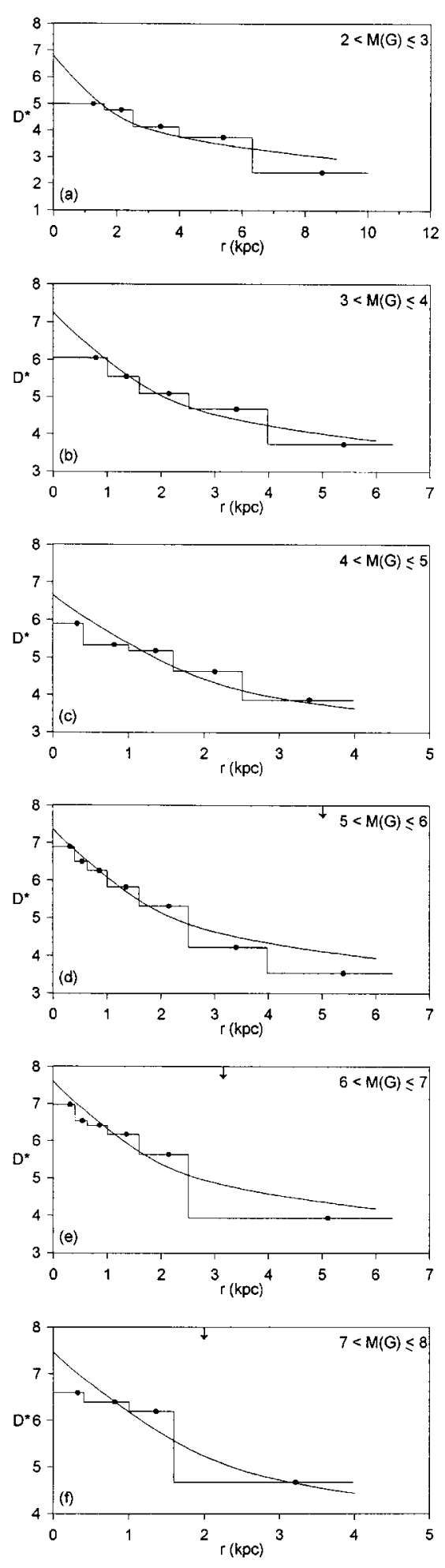

Fig. 7. Histograms of logarithmic space densities as functions of absolute magnitude for all population components combined, after assignment of turnoff- and subgiant luminosities $(2<$ $\left.M_{G} \leq 4\right)$ to a selected number of lower-metallicity class stars initially assumed to be on the main sequence $\left(5<M_{G} \leq 8\right)$. Note that, in general, the gradients now agree better with the model fits (curves), as do the corresponding extrapolated local densities (see the text)

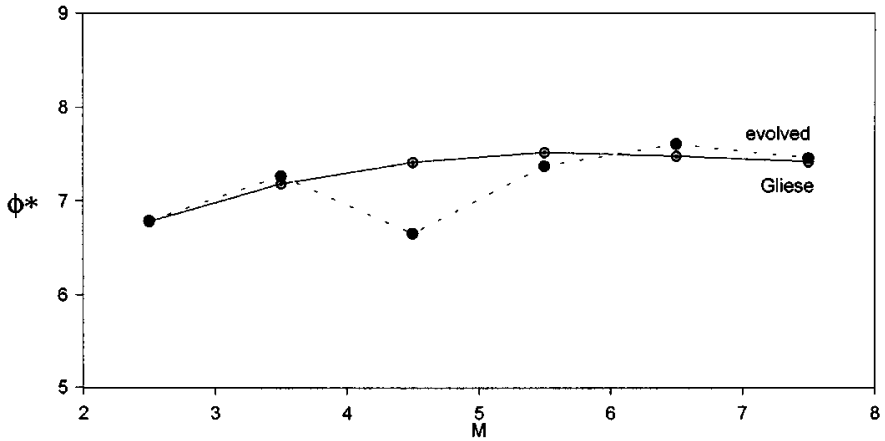

Fig. 8. Luminosity function resulting from observed local densities extrapolated in Fig. 7 (dotted line). Obviously, the Gliese standard (solid line) has been successfully used as a constraint in the first iteration of the present analysis. The disagreement at $M_{G}=4.5$ is a feature germane to old metal-poor populations (see the text)

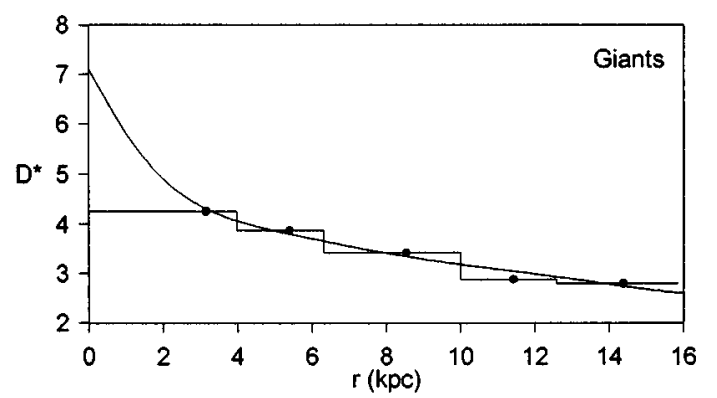

Fig. 9. Density profile for late-type population I red giants identified in the two-color diagrams of the present field. The observed gradient and the extrapolated local density agree well with the model prediction and the Gliese-Jahreiss (1992) standard, respectively (see the text)

the present data show a significant excess of intrinsically fainter stars $\left(M_{G}>6\right)$ which seems to be balanced by a strong apparent deficiency of intrinsically brighter stars $\left(M_{G}<5\right)$.

Now, inspection of Fig. 5 immediately tells us that the apparent excess of nearby stars is most likely due to the (expected) bias in the absolute magnitudes assigned to the alleged intermediate and extreme population II stars: their observed density gradients are all much steeper than predicted by the model, leading to extrapolated local densities which are too high relative to the model standard by up to more than two orders of magnitude for the fainter stars, $M_{G}>6$. Thus, the most straightforward - and, in fact, plausible - explanation is that a substantial fraction of these excess nearby stars should be redistributed to the larger distances implied by brighter absolute magnitudes, $M_{G}<4$. Also note that stars of these lowermetallicity population components which have absolute magnitudes $M_{G}<5$ cannot be main sequence stars but must be evolved to near or beyond the turnoff, even if they should have relatively young ages, $>5$ Gyr (BF90); therefore, density profiles for the brighter main sequence stars 
of these population components are naturally lacking in Fig. 5.

Thus, based on the differences between the luminosity functions of Fig. 4, and depending on the individual observed density histograms and excesses indicated in Fig. 5, between $30 \%$ and $60 \%$ (for an average of $\sim 43 \%$ ) of the relatively nearby intermediate and extreme population II stars were (randomly) selected in each of the three absolute magnitude intervals $\left(M_{G}>5\right)$ for reassignment of their $M_{G}$ in a first iteration cycle. For each star, the theoretical CMD given by BF90 was used again for substituting for the former main-sequence luminosity the brighter absolute magnitude corresponding to the more advanced evolutionary stage near the turnoff or the subgiant branch associated with its same observed $G-R$ color, $\delta_{U-G}$-derived metallicity-class, and adopted (old-)age isochrone, as in the previous cycle.

Results of this first - and only - iteration are given in Tables 1-5 and illustrated in Figs. 6-9 to show that a consistent (although not proven unique) solution can be readily found by this simple procedure. Most density histograms for the population II components derived from the alternative absolute magnitude determinations and displayed in Fig. 6 do indeed follow well the flatter predicted gradients throughout the ranges set by the completeness limits of the survey. The same also holds for the profiles combining the contributions of all three population components in Fig. 7, which yield an (extrapolated) local luminosity function (Fig. 8) that is now fully consistent with the adopted reference standard! Note that, relative to the population I stars, the local densities extrapolated from the panels of Fig. 6 for the intermediate and the extreme populations II also agree fairly well, on average, with the independent (i.e., canonical) values of $\sim 3 \%$ and $\sim 0.1 \%$, respectively (cf. Buser et al. 1998a). The large variation given in Table 5 (lines 4 and 10) for the intermediate population II at $5<M_{G}<8$ is due partly to the coarseness of the present method, partly to the intrinsic difficulty to separate these faint main sequence dwarfs from the population I red giants which occupy the same area $(G-R>\sim 1.2)$ in the two-color diagram. Still, for a total of 131 red giants (of population I) that were identified in the present sample, the density profile shown in Fig. 9 is in excellent agreement with the model prediction, and the extrapolated local density given in Table $4\left(D_{0}^{*}=7.10\right)$ again closely matches the standard $\left(D_{0}^{*}=6.92\right)$ provided by Gliese \& Jahreiss (1992). However, since the local density of red giants as derived from HIPPARCOS data may be lower by almost a factor of two (Jahreiss \& Wielen 1997), the above result has been somewhat weakened as a constraint to the present analysis.

Note also that the observed $\operatorname{dip}$ at $M_{G}=4.5$ in Fig. 8 does not disturb the agreement with the standard but is consistent with theoretical expectations: because the evolutionary tracks and isochrones become increasingly vertical near this absolute magnitude in the CMD for old metal-poor populations, the more rapid evolutionary phases away from the main sequence and through the turnoff "dilute" their original main-sequence luminosity function (i.e., IMF) into a broader absolute magnitude range which leads to significant depletion of stars at some characteristic magnitude of their present-day luminosity function, to a larger uncertainty in the determination of individual absolute magnitudes, and to accordingly modified associated Malmquist corrections (Gilmore 1984). In fact, the feature has been observed prominent in far-field stellar samples away from the Galactic plane, because due to their greater average ages relative to the normal (thin) disk, these stars also have larger scale heights resulting from their prolonged post-collapse dynamical evolution (Wielen 1974).

\section{Discussion and conclusion}

While a significant fraction of turnoff and subgiant stars were already shown to exist in the Basel fields SA51 and SA57 from the analysis of combined $R G U$ and proper motion data in the early papers by Buser \& Chiu (1981a,b), here we have demonstrated for the first time that, in the absence of proper motion data, a simple variant of the classical three-color analysis of $R G U$ observations indeed allows to derive density gradients and luminosity functions which are consistent with canonical stellar distribution functions of both the large-scale space densities and the luminosities in the local sphere of the Galaxy.

This achievement sharply contrasts with earlier results derived from the original catalogs of $R G U$ field data, which were based on Steinlin's (1968) preliminary standard of the $R G U$ system and which were shown to carry large systematic errors in the basic color and absolute magnitude calibrations (Buser 1988; Buser \& Rong 1995). In fact, using the original $R G U$ data base, density gradients and luminosity functions could not be derived consistent with both plausible density models and local luminosity functions for the large majority of Basel survey fields (e.g., Fenkart 1989a-d).

As anticipated in the introduction, the present improvements can be attributed completely to the revised standardization procedure and the extended calibrations set up for the $R G U$ system via synthetic photometry techniques. The comprehensive tie-in with the $U B V$ system then also justifies the iterative procedure adopted in Sect. 3 for determining absolute magnitudes for the lowerabundance stars by feedback from the resulting density and luminosity functions.

However, while in this paper we have been able to demonstrate in a very simple way that indeed, all the above innovations are leading to an altogether positive result, it must also be admitted that no claims of uniqueness can be made for the solution presented in Sect. 4 . 
Table 5. Local densities and their standard deviations, $D_{0}^{*} \pm \sigma_{D_{0}^{*}}$, extrapolated from observed density profiles by least-squares. The three bottom lines include the local-density differences between population components, and, for comparison, the Gliese standard

\begin{tabular}{lcccccc}
\hline$M(G)$-interval & $2-3$ & $3-4$ & $4-5$ & $5-6$ & $6-7$ & $7-8$ \\
\hline Population I & - & 7.29 & 7.24 & 7.54 & 7.56 & 6.99 \\
& - & \pm 1.56 & \pm 0.64 & \pm 0.37 & \pm 0.26 & \pm 0.19 \\
Intermediate Population II & 5.20 & 5.79 & - & 5.15 & 5.91 & 6.52 \\
& \pm 0.29 & \pm 0.27 & - & \pm 0.32 & \pm 0.16 & \pm 0.06 \\
Population II & 4.04 & 4.65 & - & 4.20 & 5.34 & 5.63 \\
& \pm 0.76 & \pm 0.59 & - & \pm 0.22 & \pm 0.11 & \pm 0.07 \\
All populations & 6.79 & 7.26 & 6.65 & 7.37 & 7.61 & 7.46 \\
& \pm 0.35 & \pm 0.19 & \pm 0.24 & \pm 0.18 & \pm 0.26 & \pm 0.33 \\
Pop. I - Intermediate Pop. II & - & 1.50 & - & 2.39 & 1.65 & 0.47 \\
Intermediate Pop II - Pop. II & 1.16 & 1.14 & - & 0.95 & 0.57 & 0.89 \\
Gliese (1969) (๑) & 6.78 & 7.18 & 7.41 & 7.52 & 7.48 & 7.42 \\
\hline
\end{tabular}

The main reason is simply that in actual practice the starby-star treatment prescribed by the classical three-color method (which was adopted here!) does not allow to account for the multiple values of the astrophysical parameters that can be associated with an individual star, given its apparent magnitude, two-color position, and an upper limit in absolute magnitude only. A fortiori, this same procedure neither allows to account for more than one or (at most!) two full iterations of propagating through the resulting density and luminosity functions the different assigned absolute magnitudes of a large sample of randomly selected stars which are scattered in a finite area of the observed $(U-G, G-R)$ two-color diagram.

Therefore, a more exacting quantitative treatment naturally requires a more performing automated analysis which also allows to exploit the full ranges of available astrophysical calibrations and constraints, such as the dependencies of the stellar colors on age, metallicity, and luminosity. Most importantly, the calibration of the observed ultraviolet excess, $\delta_{U-G}$, will need to be extended beyond the main sequence to the lower-gravity turnoff and subgiant stars in order to provide a more accurate determination of the metallicity distribution of the intermediate and extreme populations II identified in this paper. These more ambitious tasks are being completed as part of the all-survey analysis; preliminary results obtained for the first-half survey of seven fields are published in Buser et al. (1998a).

Acknowledgements. This work was supported by the Swiss National Science Foundation.

\section{References}

Becker W., 1962, Zs. f. Astrophys. 54, 155

Becker W., 1965, Zs. f. Astrophys. 62, 54
Becker W., 1980, A\&A 87, 80

Becker W., Fang Ch., 1982, A\&AS 49, 61

Buser R., 1978a, A\&A 62, 411

Buser R., 1978b, A\&A 62, 425

Buser R., 1981, in: Galaktische Struktur und Entwicklung, Buser R. (ed.) Astron. Inst. Univ. Basel, p. 31

Buser R., 1982, Mitt. Astron. Ges. 57, 233

Buser R., 1988, in Impacts des surveys du visible sur notre connaissance de la Galaxie, Fresneau A. \& Hamm M. (eds.). Comptes Rendus sur les Journées de Strasbourg, 9ème Réunion, Obs. de Strasbourg, p. 15

Buser R., Chiu L.-T.G., 1981a, in Galaktische Struktur und Entwicklung, Buser R. (ed.), Astron. Inst. Univ. Basel, p. 69

Buser R., Chiu L.-T.G., 1981b, Mitt. Astron. Ges. 52, 40

Buser R., Fenkart R.P., 1990, A\&A 239, 243 (BF90)

Buser R., Kaeser U., 1985, A\&A 145, 1

Buser R., Rong J.X., 1995, Baltic Astron. 4, 1

Buser R., Rong J.X., Karaali S., 1998a, A\&A 331, 934

Buser R., Karaali S., Topaktas L., et al., 1998b, A\&AS (in preparation)

Fenkart R.P., 1989a, A\&AS 78, 217

Fenkart R.P., 1989b, A\&AS 79, 51

Fenkart R.P., 1989c, A\&AS 80, 89

Fenkart R.P., 1989d, A\&AS 81, 187

Fenkart R.P., Karaali S., 1987, A\&AS 69, 33

Gilmore G., 1984, MNRAS 207, 223

Gilmore G., Wyse R.F.G., 1985, AJ 90, 2015

Gliese W., 1969, Veröff. Astron. Rechen-Inst. Heidelberg, No. 22

Gliese W., Jahreiss H., 1992, Third Catalogue of Nearby Stars (Preliminary Version), Astron. Rechen-Inst. Heidelberg

Jahreiss H., Wielen R., 1997, in HIPPARCOS Venice'97, Noordwijk: ESA SP-402, p. 675

Rio G. del, Fenkart R.P., 1987, A\&AS 68, 397

Sandage A., Tammann G.A., 1974, ApJ 194, 223

Steinlin U.W., 1968, Zs. f. Astrophys. 69, 276

Wielen R., 1974, in Highlights of Astronomy, Contopoulos G. (ed.), Vol. 3. Dordrecht: Reidel, p. 395

Wielen R., Jahreiss H., Krüger R., 1983, in The Nearby Stars and the Stellar Luminosity Function, Davis Philip A.G. \& Upgren A.R. (eds.). IAU Coll. 76, Schenectady, N.Y.: L. Davis Press, p. 163 